\title{
Optimización del Proceso de Atención al Cliente en un Restaurante durante Períodos de Alta Demanda
}

\author{
Rodolfo F. Schmal y Teresa Y. Olave \\ Escuela de Ingeniería en Informática Empresarial, Universidad de Talca, Talca-Chile \\ (e-mail: rschmal@utalca.cl)
}

Recibido Dic. 16, 2013; Aceptado Feb. 25, 2014; Versión final recibida Abr. 10, 2014

\begin{abstract}
Resumen
En este trabajo se aborda el problema de la insatisfacción de los clientes de un restaurante debido a los largos tiempos de atención en épocas de alta demanda. Para estos efectos se presenta una metodología de mejora en el modelo de negocios de un restaurante localizado en el puerto de San Antonio, región de Valparaíso (Chile). Para modificar la estrategia de negocio del restaurante se utilizó el modelo BMC (Business Model Canvas) y para modelar el proceso de atención al cliente se ocupó BPMN (Business Process Modeling Notation) y el diagrama SIPOC (Supplier, Input, Process, Output, Consumer). El análisis de los modelos permitió emprender acciones de mejora en el rendimiento del proceso de atención al cliente en tiempos de alta demanda, que posibilitaron una disminución en los tiempos de espera de los clientes en un $20 \%$. Se concluye que el mejoramiento propuesto ha permitido aumentar la cantidad de clientes que el restaurant es capaz de atender, lo que ha traído consigo un fuerte aumento en las ventas y en la rentabilidad del negocio.
\end{abstract}

Palabras clave: modelo de negocios, optimización, proceso de atención, restaurante

\section{Optimization of the Customer Service Process in a Restaurant during High Season Demand}

\begin{abstract}
The problem of clients' unsatisfaction in a restaurant due to the long waiting times to be served during high season is analyzed and solved. For this, a business improvement methodology of a restaurant located at the port of San Antonio, region of Valparaíso (Chile), is proposed. To modify the business strategy of the restaurant the Business Model Canvas (BMC) was employed and to model the service process the Business Process Modeling Notation (BPMN) and the diagram SIPOC (Supplier , Input , Process, Output, Consumer) were used. The analysis of the models allowed introducing actions aimed to improve the service process and reduce waiting times of customers by $20 \%$ during high season. The proposed improvement methodology has enabled a significant increase in the number of customers that the restaurant is capable of serving, bringing a strong increase in sales and profitability of the restaurant.
\end{abstract}




\section{INTRODUCCIÓN}

En los tiempos actuales, de alta competitividad, uno de los procesos que ha adquirido cada vez mayor relevancia en las organizaciones es el de atención a clientes. Para el caso de las empresas del sector gastronómico, como son los restaurantes, se trata de un proceso clave. Según Johnston y Michel (2008), Allon y Ferguson (2007) y Hui et al. (1998), los atributos que más valoran los clientes de un restaurante, son: el precio, el tiempo de atención, la localización, el ambiente y la calidad de la comida. En el mercado de los restaurantes, al igual que en otros mercados, la alternativa de modificar los precios para ajustar la oferta a la demanda, como una forma de racionar la demanda, tiende a ser más costosa que hacerlo mediante colas de espera. En este sentido se hace mención a un racionamiento eficiente cuando es preferible hacer esperar a nuevos clientes a variar el costo del menú de acuerdo a la demanda (DeGraba, 2004; Folmer y Leen, 2013). En los restaurantes, el tiempo de espera se encarga de ajustar el exceso de demanda hasta equilibrarlo con la oferta del servicio, racionando a los consumidores en vez de aumentar los precios (Agostini y Saavedra, 2008; Bose, 1996).

Este trabajo se centra en el tiempo de atención a los clientes de un restaurante, El Cholita, localizado en la región central costera de Chile, en la ciudad-puerto de San Antonio, que en su temporada alta -enero y febrero- atiende sobre 500 comensales diariamente entre las 13 y 17 horas. El restaurante posee un gran salón comedor conformado por 65 mesas con una capacidad de atención de 4 a 6 clientes por mesa y con una dotación de 14 mozos, 5 asistentes de mozos, 12 cocineros, y 2 cajeros en tiempos de alta demanda. $\mathrm{Su}$ volumen de ventas mensual, en los meses de enero y febrero, es del orden los 340 mil dólares. El restaurante posee una atractiva oferta gastronómica -caracterizada por la venta de platos con productos frescos del mar- muy demandada en la temporada alta. Sin embargo, sus prolongados tiempos de atención generan insatisfacción en los clientes. La no disponibilidad de terrenos adicionales conspira contra la posibilidad de ampliar la oferta.

Como consecuencia de lo expuesto, la capacidad de atención a los comensales del restaurante se ve ampliamente superada durante los meses de enero y febrero, y en fines de semana largos por feriados durante el resto del año. Esto se expresa en prolongados tiempos de espera de los clientes, quienes se sienten mal atendidos, lo que conlleva cancelaciones de pedidos, bajas propinas y mal trato a los mozos por parte de los clientes. Un estudio de tiempos realizado en el verano del 2012 determinó que los tiempos medios de espera eran del orden de 45 minutos desde que el cliente toma asiento y su pedido es recogido hasta que llega el primer plato, pudiendo alcanzar en algunos casos un tiempo de espera de casi una hora, y un desfase medio de tiempo entre platos de un mismo pedido de 16 minutos. La alta demanda solo puede ser encarada con una mayor rotación de clientes, solo posible con una significativa reducción en los tiempos de atención. Cabe destacar que estos problemas afectan a la totalidad de los 10 restaurantes que se encuentran en la misma zona, lo que en el largo plazo incide en una disminución en el nivel turístico de la ciudad, razón por la cual es de interés buscar alternativas de solución al problema expuesto.

El propósito de este trabajo es optimizar el proceso de atención en el restaurante para disminuir los niveles de insatisfacción de sus clientes. Los indicadores que medirán el logro de este propósito son el tiempo de disminución de los tiempos de espera por parte de los clientes y de los tiempos de desfase en las entregas de los platos contenidos en un mismo pedido. En términos de metas, se propuso para la temporada veraniega del 2013 una disminución del 20\% en los tiempos actuales de espera, y una reducción en un 40\% del tiempo que transcurre desde que se entrega el primer y último plato de un mismo pedido. Para el logro del objetivo especificado se procedió a identificar el modelo del negocio en el que se desenvuelve el restaurante, tal cual es y tal cual se aspira que sea, seguido por un análisis de sus procesos clave, centrándose este trabajo en uno de ellos, el de atención al cliente. Como resultado de este análisis, surgió una propuesta orientada a disminuir los tiempos de atención de los clientes que recoge las buenas prácticas del sector y la experiencia existente de aplicación de tecnologías de información y comunicación en el sector gastronómico.

\section{MODELADO DEL NEGOCIO Y DEL PROCESO DE ATENCIÓN}

El restaurante en estudio se caracteriza por su naturaleza familiar, su oferta de productos marinos frescos y un modo de gestión convencional basado en la experiencia de sus propietarios. En ninguno de estos restaurantes localizados en el mismo sector se hace mayor uso de las potencialidades que ofrecen las tecnologías de información y comunicación (TICs) disponibles en el mercado para apoyar los procesos de atención a los clientes. A pesar de que las TICs se han expandido a todos los ámbitos de la vida diaria, solo en los últimos años ha logrado introducirse, tímidamente, en el sector gastronómico. Esta incursión se ha dado especialmente en algunas de las etapas de los procesos de atención a los clientes de los restaurantes, tanto de consumo masivo como en los de alta cocina. Esta incursión de las TICs se ha dado en las etapas de recepción de pedidos y/o reservas de mesas, compras de ingredientes, facturación de 
pedidos, confección de menús y/o verificación de disponibilidad de platos dentro de los menús (Pieskä et al., 2013). Un estudio en torno al sector revela que los restaurantes consolidados cuya demanda es de alta variabilidad, con elevados niveles de incerteza, y que dependen fuertemente de días festivos y de las condiciones climáticas, tienden a disponer de sistemas de gestión centralizados apoyados por TICs (Pornthipa y Kessuvan, 2013).

Los fundamentos de la aplicación de las TICs en las distintas etapas del proceso de atención a los clientes se centran en facilitar la recepción de los pedidos y los cambios de menús, optimizar el reaprovisionamiento de ingredientes, mejorar la calidad del servicio, disminuir los tiempos de atención, e incrementar la información nutritiva de los menús ofrecidos. A pesar de la introducción de las TICs en los restaurantes, el proceso de atención sigue centrado en el cliente donde seguirá importando fuertemente la interacción humana y el servicio personalizado, especialmente en aquellos locales no pertenecientes a cadenas de restaurantes. Entre las TICs que se han aplicado en los restaurantes y que apuntan a optimizar los procesos de atención a clientes mediante el uso de computadoras, destacan: la identificación por frecuencias de radio (Radio Frecuency Identification -RFID-) junto con los asistentes digitales personales (PDA), que han estado siendo superados por los tablets y/o los menús o pantallas en mesa, que en algunos casos es complementado con servicios de robots capaces de servir los pedidos efectuados por los comensales (Pieská et al., 2013).

Los menús en mesa son ocupados por los propios comensales para realizar sus pedidos digitalizando sus opciones vía pantallas disponibles en las mesas que ocupan. Bajo esta tecnología los mozos son liberados de recibir pedidos, limitándose a servir los pedidos efectuados por los comensales. En la modalidad de tablets, cada mozo tiene un tablet para digitalizar los pedidos que realicen los comensales. Esta última opción fue la seleccionada en consideración al perfil de los clientes (comensales), de nivel socioeconómico medio y un bajo nivel de alfabetización tecnológica.

Tras las alternativas tecnológicas mencionadas, en el mercado nacional existen diversos sistemas de software orientados a la administración de restaurantes. De ellos, se escogió el software Restbar, decisión sustentada en: a) su capacidad para integrar prestaciones de servicio (control y reposición de inventarios, facturación de mesas, generación de menús, elaboración de estadísticas de ventas y costos); b) sus bajos requerimientos tecnológicos y de capacitación; c) satisfacer los requerimientos de no ocupar la red de internet planteados por los propietarios, y d) su bajo costo en relación a otras alternativas. Esta selección se vio reforzada por su exitosa implantación en restaurantes nacionales, tales como los restaurantes La Pesca localizados de La Dehesa, Vitacura y Los Dominicos, Bakulic de La Serena, Logroño de San Antonio, Santo Visto de Rancagua, Zutto Sushi de Concepción, Bendita de Pucón, Entre Amigos de Osorno, Club de Golf de Rocas Santo Domingo.

El desarrollo del trabajo se inició con el levantamiento del modelo de negocio existente ("as is") del restaurante utilizándose como herramienta el Business Model Canvas (BMC). Esta es una herramienta conceptual que contiene los componentes esenciales para expresar la lógica del negocio de una empresa específica mediante un lienzo con 9 componentes (Osterwalder y Pigneur, 2010; Márquez, 2010). En relación con otras herramientas similares (Software Ecosystem Model y el Board of Innovation), el uso de BMC se fundamenta por su capacidad para capturar y comunicar visualmente, en forma eficaz y eficiente, la propuesta de valor de una empresa a sus clientes y la arquitectura bajo la cual se estructura (Lucassen et al., 2012). Luego se procedió a modelar el negocio tal cual los dueños aspiran que sea ("as to be"), identificando los componentes que se desean modificar y las acciones a desarrollar para que se lleven a cabo. Éstas se obtuvieron, al igual que en la etapa anterior, a partir de reuniones de trabajo sostenidas con ejecutivos del restaurante.

Posteriormente se representó el proceso clave de atención al cliente tal cual se lleva en la actualidad, en base a técnicas asociadas al flujo de datos (Sanchis et al., 2009; Schmal et al., 2010) y ocupando la herramienta de modelamiento de procesos Bizagi (Bizagi, 2012) que se apoya en la notación de BPMN (Business Process Modeling Notation) (White y Miers, 2008; Freund et al., 2012). BPMN es una notación gráfica destinada a modelar un proceso de negocios por la vía de describir la lógica imperante en su interior. Su selección se explica por tratarse de un estándar reconocido internacionalmente y por su capacidad para representar en forma simple los distintos elementos que participan en los procesos. BPMN se complementó con el uso del diagrama SIPOC (Supplier, Input, Process, Output, Consumer) de la empresa Sigma (Nold, 2011) por su contribución a la identificación de las entradas a los procesos y sus proveedores, junto a las salidas y sus consumidores. Finalmente, se propuso un nuevo modelo a partir del rediseño del proceso de atención que incluyó un análisis de sus fortalezas, oportunidades, debilidades y amenazas (FODA) (Jonassen, 2012), de las buenas prácticas existentes en el sector gastronómico, y de las potencialidades que ofrecen las tecnologías de información y comunicación capaces de romper los paradigmas imperantes. 


\section{RESULTADOS}

Como resultado de las reuniones de trabajo llevadas a cabo con ejecutivos y personal del restaurante, se obtuvo una representación del modelo del negocio existente (Tabla 1), siguiendo el BMC de Osterwalder y Pigneur.

Tabla 1: Modelo del negocio actual

\begin{tabular}{|c|c|c|c|c|}
\hline \multirow[t]{2}{*}{$\begin{array}{l}\text { Alianzas } \\
\text { estratégicas } \\
\text { Pescadores } \\
\text { de la zona }\end{array}$} & $\begin{array}{l}\text { Actividades claves } \\
\text { Captación de clientes; } \\
\text { Atención al cliente; } \\
\text { Elaboración de platos; } \\
\text { Compra de ingredientes }\end{array}$ & \multirow[t]{2}{*}{$\begin{array}{l}\text { Propuesta de } \\
\text { valor } \\
\text { Servicio } \\
\text { gastronómico } \\
\text { centrado en } \\
\text { productos } \\
\text { marinos frescos } \\
\text { con vista al mar }\end{array}$} & $\begin{array}{l}\text { Relaciones con clientes } \\
\text { Pisco sour de cortesía; } \\
\text { Atención personalizada; } \\
\text { Facilidades de pago (efectivo, } \\
\text { cheques, tarjetas bancarias) }\end{array}$ & \multirow[t]{2}{*}{$\begin{array}{l}\text { Segmentos de } \\
\text { clientes } \\
\text { Turistas de clase } \\
\text { media que visitan } \\
\text { el puerto de San } \\
\text { Antonio } \\
\text { procedentes de } \\
\text { Santiago }\end{array}$} \\
\hline & $\begin{array}{l}\text { Recursos claves } \\
\text { Personal; Productos } \\
\text { marinos y otros; } \\
\text { Localización }\end{array}$ & & $\begin{array}{l}\text { Canales de distribución } \\
\text { Venta directa en local; } \\
\text { Promoción vía mozos }\end{array}$ & \\
\hline \multicolumn{2}{|c|}{$\begin{array}{l}\text { Estructura de costos } \\
\text { Sueldos }(30 \%) \text {, insumos }(35 \%) \text { y otros }(5 \%)\end{array}$} & \multicolumn{3}{|c|}{$\begin{array}{l}\text { Flujo de ingresos } \\
\text { Ventas de servicios gastronómicos concentradas en } \\
\text { temporadas altas (100\%) }\end{array}$} \\
\hline
\end{tabular}

El análisis del modelo del negocio "as is" (como es actualmente) permitió identificar dos problemas: la ineficiente atención al cliente en temporadas altas y la escasa cantidad de clientes en temporadas bajas. En consecuencia, un nuevo modelo de negocio "as to be" (como debe ser) deberá apuntar a desestacionalizar la demanda, lo que implica realizar gestiones conducentes a elevar la demanda en temporadas bajas, ampliando sus servicios gastronómicos a empresas y promoviendo descuentos a agencias de turismo; y abordar los procesos de atención al cliente y de elaboración de platos con apoyo de las TICs. En la tabla 2 se presenta el modelo del negocio al que se aspira.

Tabla. 2: Modelo del negocio deseado

\begin{tabular}{|c|c|c|c|c|}
\hline \multirow[t]{2}{*}{$\begin{array}{l}\text { Alianzas } \\
\text { estratégicas } \\
\text { Pescadores, } \\
\text { Agencias de } \\
\text { turismo, } \\
\text { Empresas de } \\
\text { San Antonio y } \\
\text { de la región }\end{array}$} & $\begin{array}{l}\text { Actividades claves } \\
\text { Captación de clientes y } \\
\text { empresas; Atención al } \\
\text { cliente; Elaboración de } \\
\text { platos; } \\
\text { Compra de ingredientes; } \\
\text { Capacitación de personal; } \\
\text { Automatización del } \\
\text { proceso de gestión del } \\
\text { restaurante }\end{array}$ & \multirow[t]{2}{*}{$\begin{array}{l}\text { Propuesta de } \\
\text { valor } \\
\text { Ágil Servicio } \\
\text { gastronómico } \\
\text { centrado en } \\
\text { productos } \\
\text { marinos frescos } \\
\text { con vista al mar }\end{array}$} & $\begin{array}{l}\text { Relaciones con clientes } \\
\text { Pisco sour de cortesía; } \\
\text { Atención } \\
\text { personalizada; } \\
\text { Facilidades de pago } \\
\text { (efectivo, cheques, } \\
\text { tarjetas bancarias); } \\
\text { Descuentos especiales } \\
\text { para familias y } \\
\text { empresas fuera de } \\
\text { temporadas altas }\end{array}$ & \multirow[t]{2}{*}{$\begin{array}{l}\text { Segmentos de } \\
\text { clientes } \\
\text { Turistas de clase } \\
\text { media que visitan el } \\
\text { puerto de San } \\
\text { Antonio procedentes } \\
\text { de Santiago } \\
\text { Empresas que } \\
\text { organicen eventos } \\
\text { (almuerzos, cenas } \\
\text { de aniversario, etc.) }\end{array}$} \\
\hline & $\begin{array}{l}\text { Recursos claves } \\
\text { Personal; Productos } \\
\text { marinos y otros; } \\
\text { Localización; TICs; } \\
\text { Promotores p/ captación } \\
\text { de empresas }\end{array}$ & & $\begin{array}{l}\text { Canales de distribución } \\
\text { Venta directa en local; } \\
\text { Promoción radial, vía } \\
\text { web, etc. }\end{array}$ & \\
\hline \multicolumn{2}{|c|}{$\begin{array}{l}\text { Estructura de costos } \\
\text { Sueldos }(30 \%) \text {, tecnologías (10\%) insumos } \\
(35 \%) \text { y otros (5\%) }\end{array}$} & \multicolumn{3}{|c|}{$\begin{array}{l}\text { Flujo de ingresos } \\
\text { Ventas de servicios gastronómicos: en temporadas altas, } 80 \% \text {; } \\
\text { en temporadas bajas, } 20 \% \text { ) }\end{array}$} \\
\hline
\end{tabular}

Teniendo identificados el modelo del negocio existente y el que se aspira tener, se elaboró una primera representación del proceso de atención al cliente, tal cual estaba siendo desarrollado (figura 1), la que posteriormente se llevó a la notación BPMN de Bizagi (figura 2).

Como consecuencia del análisis efectuado, se elaboró el diagrama SIPOC (Tabla 3) que permite identificar los proveedores, las entradas, las actividades, salidas y clientes involucrados en el proceso de atención al cliente. 


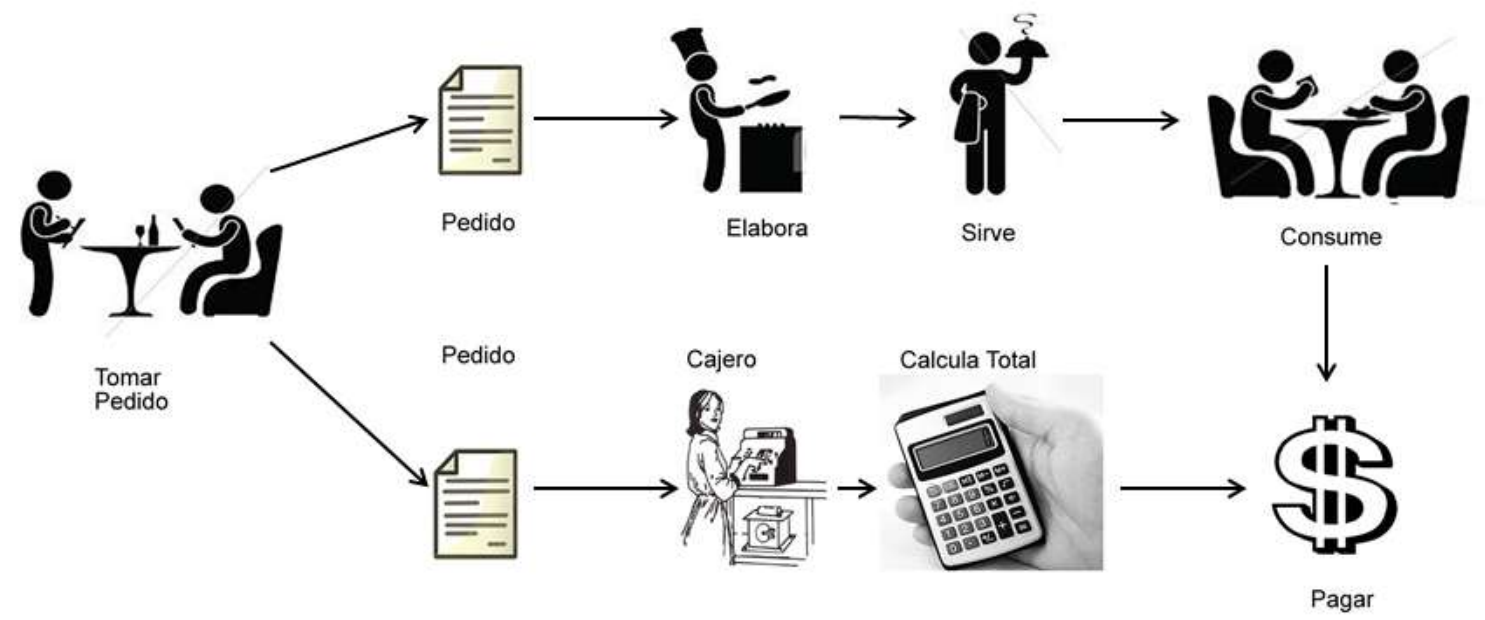

Fig. 1: Proceso actual de atención al cliente

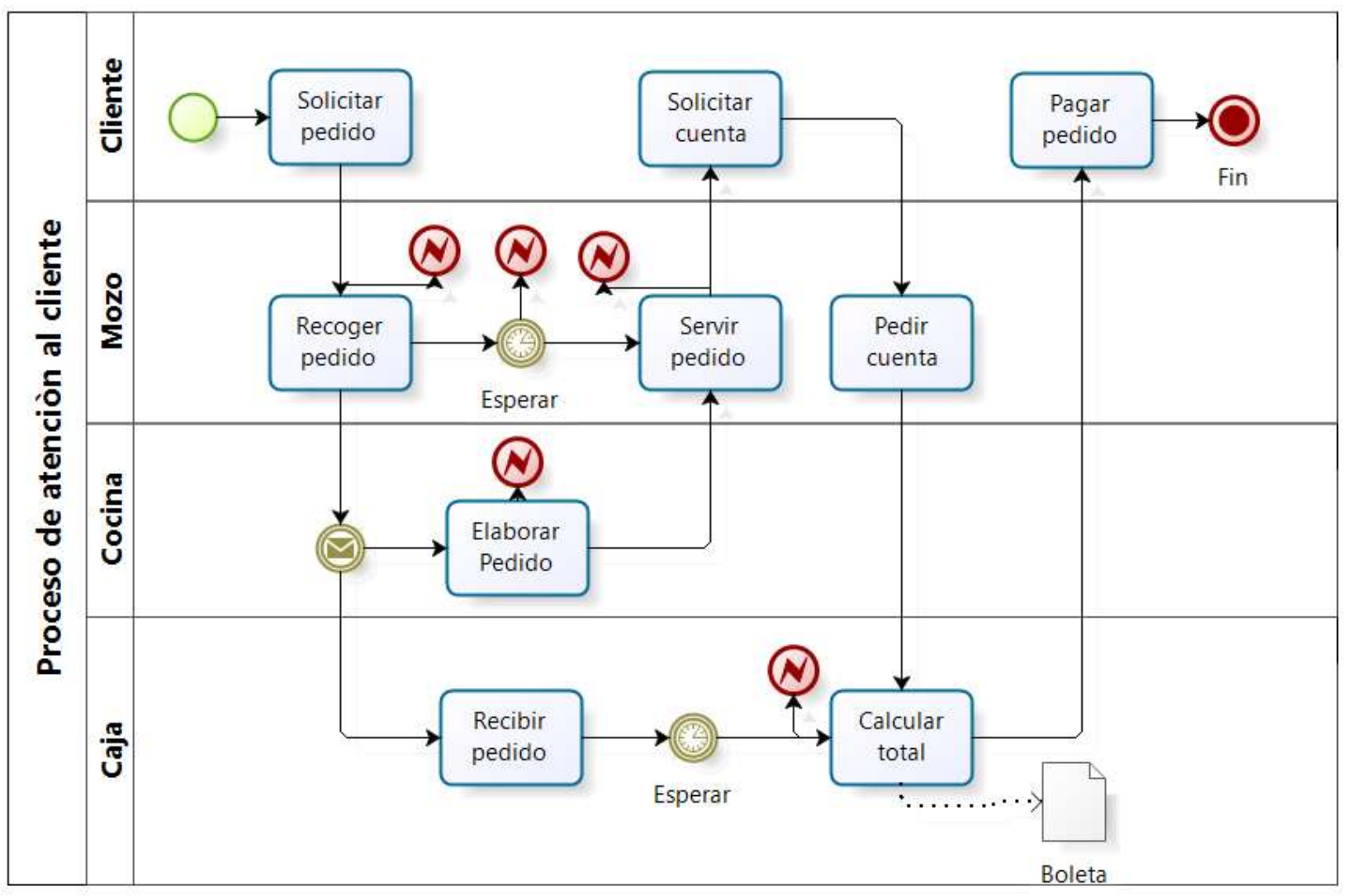

Fig. 2: Modelo actual del proceso de atención al cliente actual en BPMN

Tabla 3: Diagrama SIPOC del proceso de atención al cliente

\begin{tabular}{|l|l|l|l|l|}
\hline \multicolumn{1}{|c|}{ S } & \multicolumn{1}{c|}{ I } & \multicolumn{1}{c|}{ P } & \multicolumn{1}{c|}{ O } \\
\hline Cliente & Carta, selección & Registrar pedido & Pedido registrado & Chef \\
\hline Chef & Pedido registrado & Elaborar pedido & Pedido elaborado & Mozo \\
\hline Mozo & Pedido elaborado & Servir pedido & Pedido servido & Cliente \\
\hline Cliente & Pedido servido, solicitud de cuenta & Cobrar pedido & Pedido cobrado, valor cuenta & $\begin{array}{l}\text { Cajero, } \\
\text { Cliente }\end{array}$ \\
\hline $\begin{array}{l}\text { Cajero, } \\
\text { Cliente }\end{array}$ & Pedido cobrado, cuenta & Pagar pedido & Pedido pagado, boleta, vuelto & Cliente \\
\hline
\end{tabular}


Los indicadores clave de desempeño, KPI (Setijono y Dahlgaard, 2007), que se ocuparon, tanto de entrada, como del proceso y de salida que permitan cuantificar el nivel de logro de los cambios que más adelante se proponen, son los que aparecen en la Tabla 4.

Tabla 4: KPI Proceso de Atención a Cliente

\begin{tabular}{|l|l|}
\hline \multicolumn{1}{|c|}{ KPI } & \multicolumn{1}{c|}{ Descripción } \\
\hline de entrada & $\begin{array}{l}\text { Tasa de clientes diarios por mesa } \\
\text { Tasa de mozos por cliente }\end{array}$ \\
\hline de proceso & $\begin{array}{l}\text { Tasa de elaboración = tiempo de elaboración/tiempo total de atención } \\
\text { Tasa de atención = Tiempo de atención/tiempo de permanencia en local }\end{array}$ \\
\hline de salida & $\begin{array}{l}\text { Tasa de satisfacción = total de clientes con reclamos/total de clientes } \\
\text { Tasa de circulación = total de pedidos atendidos por mesa } \\
\text { Promedio de ingresos por cliente }\end{array}$ \\
\hline
\end{tabular}

Entre las debilidades identificadas en el proceso, que elevan los tiempos de atención al cliente, destacaron las siguientes: 1. La letra ilegible con que los mozos recogen los pedidos, provocando errores y generando confusión, tanto en caja para los efectos de los cobros como en la cocina para la elaboración de los platos; 2. Los clientes no conocen el detalle de la cuenta que se les está cobrando; 3 . No existe comunicación entre mozos y el personal de cocina en torno a la disponibilidad de los platos que figuran en el menú; 4. Sobrecarga de trabajo por parte del cajero, quien tiene la responsabilidad de sumar los valores contenidos en los pedidos, generar las boletas de cobro, recibir dinero y entregar vuelto; y 5 . Dificultades para cuadrar la caja al final del día.

En consideración a las debilidades encontradas, junto con el análisis de las buenas prácticas imperantes en los restaurantes y las tecnologías disponibles se rediseñó el proceso sobre la base de: ampliar las dependencias de la cocina; anticipar el procesamiento de algunos alimentos (limpieza de pescados y mariscos, desmenuzamiento de verduras, preparación de ensaladas, elaboración de empanadas); reponer oportunamente los ingredientes más requeridos; e incorporar un software de apoyo a la gestión de los comedores, de la caja y del inventario de insumos.

La representación y el modelamiento de este rediseño se muestran en las figuras 3 y 4.

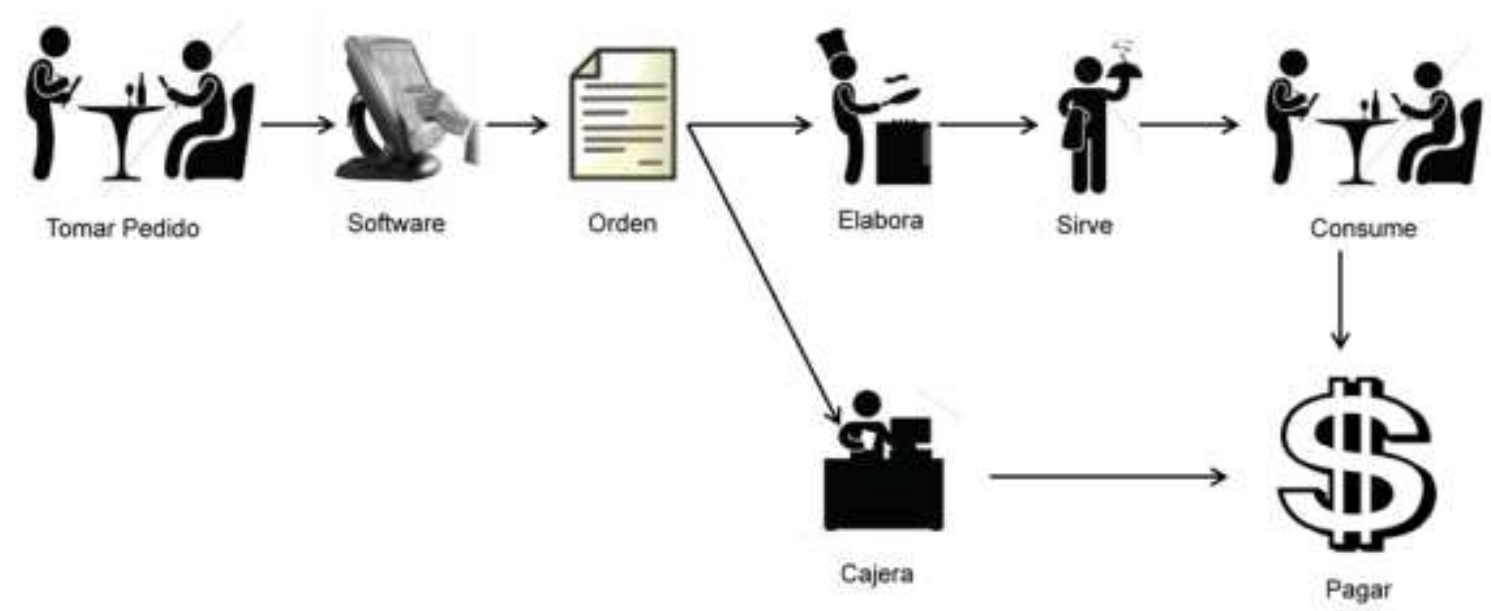

Fig. 3: Proceso de atención al cliente deseado

De las tecnologías disponibles en el mercado para la gestión de restaurantes (Díaz de Orbe, 2010), se evaluaron las pantallas y los botones en mesa, junto con los tablets. En cuanto al software para la gestión de restaurantes, se evaluaron los sistemas Gour-net (http://www.gour-net.cl), Restbar (http://www.restbar.com, Ambit Technology, 2011), Datahouse Company (http://www.datahouse company.com) y SoftRestaurant (http://www.softrestaurant.com), seleccionándose la tecnología de los tablets para ser utilizados por los mozos, y el software Restbar, por responder a los requerimientos planteados por el restaurante, en particular, no demandar el uso de internet por el riesgo que conlleva una eventual caída en las comunicaciones, cuyas consecuencias en tiempos de alta demanda no son admisibles. Este software, desde su instalación, implementación y prueba a fines del año 2012, ha ayudado de una manera significativa al funcionamiento interno del restaurante, mejorando la comunicación entre las distintas áreas de la empresa y el control sobre el inventario de los ingredientes, lo que contribuye a su oportuna reposición. 


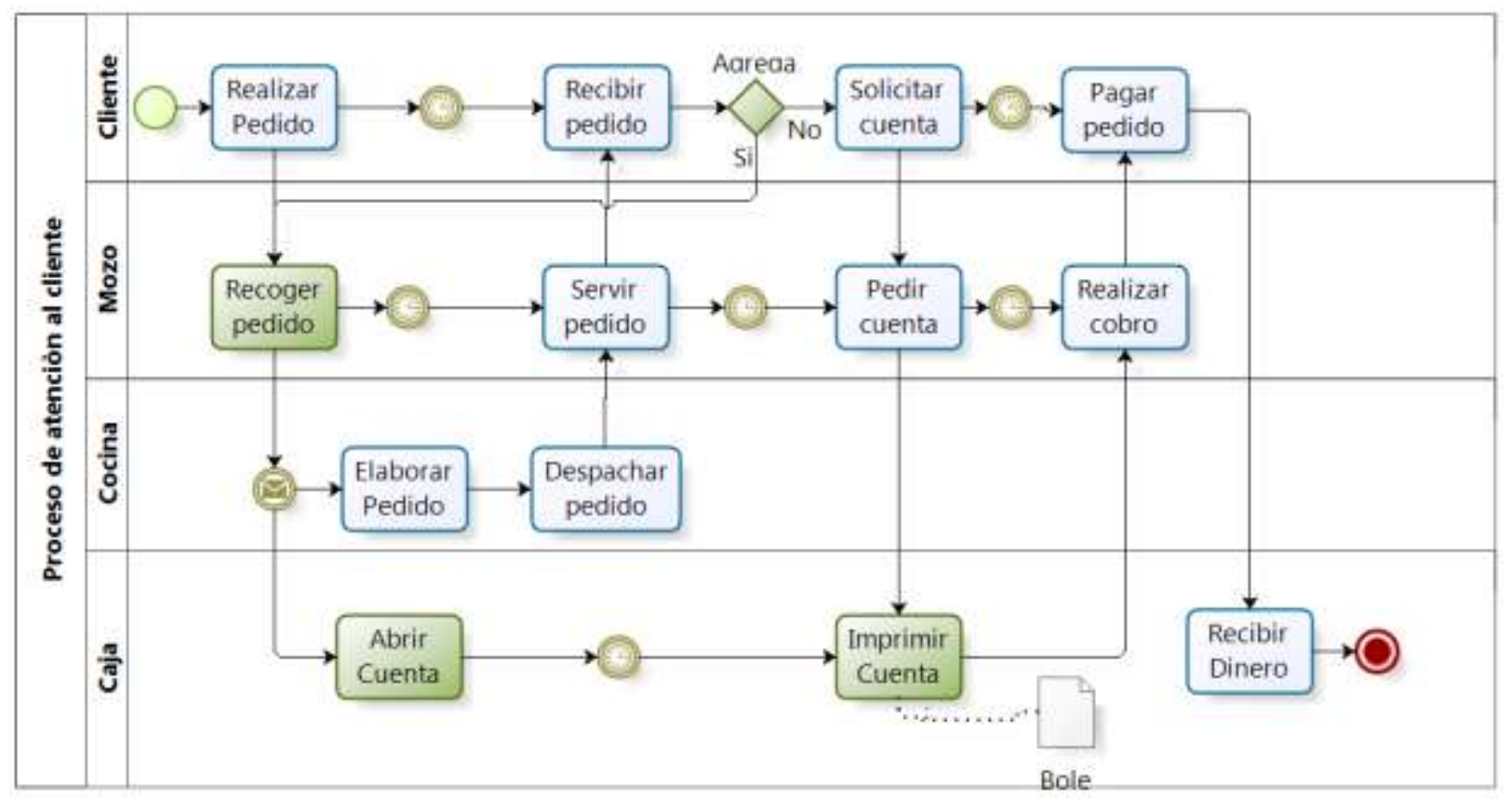

Fig. 4: Modelo propuesto para el proceso de atención al cliente en BPMN

Lo propuesto tuvo su primera prueba de fuego fueron los meses de enero y febrero del año 2013, meses de temporada alta. Tal como se aspiraba, del rediseño planteado y la incorporación del software de apoyo a la gestión, se logró que: a) dejaran de existir pedidos ilegibles, evitando errores y confusiones, tanto por parte de los mozos, cajeros como cocineros; b) los clientes conocieran el detalle de los cobros asociados a los pedidos; c) los cajeros se limitaran a recibir pagos e imprimir comprobantes de pago y vean facilitada su función de cuadrar la caja; d) los mozos conocieran anticipadamente la disponibilidad de platos que figuran en el menú; y e) los cocineros alertaran oportunamente respecto de los insumos con bajo nivel de inventarios y que requieren reponerse.

La incorporación del software fue complementada con la ampliación de la sala de la cocina, la que ayudó a que disminuyeran los tiempos de espera en la elaboración de los pedidos; y se pudieran anticipar actividades vinculadas al procesamiento de los alimentos (limpieza de pescados y mariscos, desmenuzamiento de verduras, preparación de ensaladas, elaboración de empanadas). Por otra parte, la reposición oportuna de inventarios de los ingredientes de los distintos platos posibilitó disminuir significativamente la no disponibilidad de platos ofrecidos en el menú y que son demandados por los clientes. Como consecuencia de los cambios impulsados, los tiempos medios de espera en los meses de alta demanda, han disminuido de 45 a 30 minutos, y los tiempos medios de desfase entre platos de un mismo pedido descendieron de 16 a 6 minutos.

\section{CONCLUSIONES}

Se concluye que el rediseño del proceso de atención al cliente, con el apoyo de un software de apoyo a la gestión, la ampliación de la sala de cocina y la reposición anticipada de inventarios, ha logrado elevar significativamente el nivel de satisfacción de los clientes, transformando al restaurante en un referente para sus competidores, consolidándose económicamente.

Los resultados alcanzados a la fecha estimulan al restaurante a avanzar en la senda de la incorporación de TICs para apoyar su gestión con miras a tener clientes satisfechos no solo con la calidad de los platos ofrecidos, sino con los tiempos de atención. Es importante señalar que las restricciones de espacio existentes, impiden ampliar el restaurante, razón por la cual se hace necesario implementar una política de mejoramiento continuo. Esta política deberá incluir incentivos para la capacitación del personal, tanto en materia de atención a clientes como en el aprovechamiento de las oportunidades que brindan las tecnologías para apoyar el trabajo que realizan.

Por otra parte, con la extensión de internet y los mayores niveles de seguridad en las comunicaciones, con el tiempo se proyecta que los clientes tengan un perfil de menor resistencia al uso de las TICs. Por ello, en el corto plazo se aspira incorporar servicios adicionales que en la actualidad el restaurante no proporciona, como es el de la reserva de mesas vía internet. 


\section{REFERENCIAS}

Agostini, C. y Saavedra, E. , Racionamiento Eficiente en una Facilidad Esencial: El Caso del Puerto de San Antonio (en línea), 2008.

http://www.tdlc.cl/DocumentosMultiples/Informe_Claudio_Agostini_y_Eduardo\%20Saavedra_C_136_07.pdf. Acceso: 18 de octubre (2013).

Allon, G. y Federgruen, A., Competition in Service Industries, Operation Research, 55(1), 37-55 (2007).

Ambit Technology, Manual de Usuario Software RestBar (en línea), 2011.

http://www.restbar.com/descargas/ManualDeRestBar.pdf. Acceso: 10 de julio (2013).

Bizagi, Bizagi Process Modeler (en línea), 2012.

http://www.bizagi.com/esp/descargas/BPMNbyExample.pdf. Acceso: 13 de marzo (2013).

Bose, P., Adverse selection. Waiting Lists and Restaurant Rationing, International Journal of Industrial Organization, 15, 335-347 (1996).

DeGraba, P., Reconciling the off-net pricing principle with efficient network utilization, Information Economics and Policy, 16(3), 475-494 (2004).

Díaz de Orbe, G., Sistema Integral para la Gestión de Restaurantes, Proyecto de Título Escuela Técnica Superior de Ingeniería, Universidad Pontificia de Comillas, España ( 2010).

Folmer, H. y Leen, A., Why do successful restaurants not raise their prices?. Letters in Spatial and Resource Sciences, 6(2), 81-90 (2013).

Freund, J., Rucker, B. y Hitpass, B., BPMN 2.0 Manual de Referencia y Guía Práctica, Depto. Informática, Universidad Técnica Federico Santa María. Stgo-Chile (2012).

Hui, M., Thakor, M. y Gill, R., The Effect of Delay Type and Service Stage on Customers' Reactions to Waiting, Journal of Consumer Research, 24(4), 469-80 (1998).

Jonassen, D.H., Designing for decision making, Education Tech Research Dev, 60(2), 341-359 (2012).

Johnston R. y Michel, S., Three outcomes of service recovery: Customer recovery, process recovery and employee recovery. International Journal of Operations \& Production Management. 28(1), 79-99 (2008).

Lucassen, G., Brinkkemper, S., Jansen, S. y Handovo, E., Comparison of Visual Business Modeling Techniques for Software Companies. Lecture Notes in Business Information Processing, 114, 79-93, (2012).

Márquez, J.F., Innovación en Modelos de Negocio: la metodología de Osterwalder en la práctica. Revista MBA EAFIT, 1, 30-47, (2010).

Nold H., Merging Knowledge Creation Theory with the Six-Sigma Model for Improving Organizations: The Continuous Loop Model. International Journal of Management, 28(2), 469-477 (2011).

Osterwalder, A. y Pigneur, Y., Business Model Generation, John Wiley \& Sons. 288 pp. (2010).

Pieskä, S., Liuska, M., Jauhiainen, J. y Auno A., Intelligent Restaurant System Smartmenu. $4^{\text {th }}$ IEEE International Conference on Cognitive Infocommunications, Budapest - Hungría, 2 al 5 de diciembre (2013).

Pornthipa, O. y Kessuvan, A., A Study of Large Scale Food Services Best Practices in Thailand: A Case Study of HORECAs, 10th IEEE International Conference on Service Systems and Service Management, Hong Kong - China, 17 al 19 de julio (2013).

Sanchis, R., Poler, R. y Ortiz, A., Técnicas para el Modelado de Procesos de Negocio en Cadenas de Suministro, Información Tecnológica, 20(2), 29-40 (2009).

Schmal, R., López, S., Cabrales, F. y Acuña, D., Modelado de Procesos de Negocio para la Gestión de Patentes en Universidades, Información Tecnológica, 21(6), 113-124 (2010).

Setijono D. y Dahlgaard J., Customer value as a key performance indicator (KPI) and a key improvement indicator (KII), Measuring Business Excellence, 11(2), 44-61 (2007).

White, S.A. y Miers D., BPMN Modeling and reference guide, Future Strategies Inc. Home, USA. 231 pp. (2008). 